\title{
Are we ready to use nature gardens to treat stress-related illnesses?†
}

\author{
Peter A. Coventry and Piran C.L. White
}

\section{Summary}

In this issue, Stigsdotter et al show that nature gardens offer similar benefits to cognitive-behavioural therapy for managing stress-related illnesses among people on sick leave. There is scope for pragmatic trials to establish the processes involved and highlight the co-benefits that nature gardens offer for health and the environment.

\section{Declaration of interest}

None.

\section{Copyright and usage}

(c) The Royal College of Psychiatrists 2018
Peter Coventry is a health services researcher based at the Department of Health Sciences, University of York, UK, whose research focuses on evaluating interventions to improve health and well-being in people with common mental health problems. Piran White is based at the Environment Department, University of York, UK, and conducts interdisciplinary research on human-environment interactions, including the services and benefits we derive from nature and the links between ecosystem and human health.

Adjustment disorders and reactions to severe stress are more typic ally recognisable as stress-related illnesses and are synonymous with subthreshold depression and anxiety, burnout or emotional distress These disorders are typically brought about by exposure to severe or continued stress - for example, by the onset of a life event - and are characterised by impaired mood, anxiety and/or worry, reduced attention and problems of social functioning. In primary care settings, stress is reported in over half of working-age adults, especially among women, and symptoms of burnout and exhaustion are common. Those with higher levels of perceived stress also commonly report depressive and anxiety symptoms. ${ }^{1}$

There are no guideline recommendations on how to treat stressrelated illnesses. There is little evidence for using psychological therapies, such as cognitive-behavioural therapy (CBT), to support the return to work of people on sick leave because of adjustment disorders. ${ }^{2}$ Additionally, CBT is a complex intervention whose effectiveness is reliant on the skill of practitioners and waiting times remain a barrier to accessing treatment. In this context, there is an emerging consensus that we need new care models that involve using placebased interventions to deliver integrated solutions for the improvement and promotion of mental and physical health. ${ }^{3}$ There is a growing recognition of the mental and physical health benefits of exposure to the natural environment at the population and individual level. These benefits can be significantly greater for lower income groups, ${ }^{4}$ but studies in the UK and the US highlight income, proximity and availability of transport as key barriers to the use of green spaces; these combine to militate against the use of green spaces by the groups in society which may benefit most. Moreover, declining central and local government funds have led to a reduction in spending on green space. In addition to health and well-being benefits, green spaces may also provide co-benefits such as reducing socioeconomic health inequalities, contributing to climate change mitigation, reducing pollutant levels and enhancing biodiversity. Ensuring the availability of high quality, accessible, natural spaces

† See pp. 404-411, this issue. close to where people live and work is one of the targets set out in the UK Government's 25-year plan for the environment. ${ }^{5}$ Against a background of decreasing public spending, meeting this target will likely require new models for investment in green-space provision and management.

\section{Findings from previous research}

There is some evidence from crossover and controlled trials that physical activity in green spaces confers greater benefits for wellbeing and possibly attention than comparable activity in built environments. ${ }^{6}$ Similarly, a self-paced 30 min walk in a natural environment that included parkland or a canal path with vegetation has been shown to afford greater restoration experiences and improved cognitive function than a comparable walk in a pleasant, urban-residential setting, even $30 \mathrm{~min}$ after leaving the environment. ${ }^{7}$ Over and above the salutogenic effects of walking, there is a theoretical basis drawn from environmental psychology for assuming that, unlike synthetic environments, natural environments have particular qualities that promote health and well-being. Critical here is attention restoration theory, which suggests that natural environments offer experiences and opportunities to indulge in activities that attract our involuntary attention and fascination and thereby eliminate directed attention fatigue, restore concentration and reduce stress. There is mixed evidence from controlled and observational studies that horticultural therapy - which involves horticultural activities with plants and nature under the supervision of trained professionals and is delivered either in isolation or in combination with other forms of non-pharmacological therapy - can have positive effects on mood, addiction, frequency of negative thoughts and psychoticism. ${ }^{8}$ However, much of this evidence is drawn from highly selected and heterogeneous clinical populations and is of low quality. An alternative approach rests on the idea that natural environments might confer greater health benefits if they are modified to maximise opportunities to engage with nature. Of particular interest here are the use of nature gardens that have been specifically designed to enhance and encourage outdoor activity. There are no previous controlled trials of nature gardens in the general adult population or in clinical populations. However, White et $a l^{9}$ have recently shown that time spent outdoors in a purposely renovated garden, with active and passive spaces and a rich sensory environment that included fruit trees and vegetable beds, was positively associated with improvements in mood in residential dementia patients. Furthermore, this study showed that this association was time dependent, with improvements in mood occurring after just 20 min and peaking at a duration of 80-90 min, suggesting 
there is both a minimal and a maximal time to be spent outdoors in nature to accrue health benefits.

\section{Findings from Stigsdotter et al}

In this issue, Stigsdotter et al describe their findings from a randomised controlled trial of the efficacy of a nature-based intervention that included a bespoke nature garden (known as the Nacadia ${ }^{\circ}$ therapy garden) for adults with stress-related illnesses. Participants aged 20-60 years old with psychiatric diagnoses of adjustment disorder or reactions to severe stress (but excluding post-traumatic stress disorder) and incapable of working for at least 3 months were recruited into the study by health practitioners and insurance companies in the municipality of Hørsholm in Denmark. Interventions were either a 10 -week programme of Nacadia ${ }^{\star}$ nature-based therapy (NNBT) - which included individualised and guided nature-based activities (e.g. gardening), therapeutic conversations based on CBT and psychoeducation based on mindfulness-based stress reduction - or a 10-week manualised programme of hourly CBT validated for use with people with stressrelated illnesses. NNBT also included reflection and relaxation time and physical and mental awareness exercises to foster mindful, non-judgemental awareness of nature experiences in the attentional field and homework practice. Importantly, this trial was a test of equivalence between NNBT and CBT and the primary outcome was a self-rated measure of perceived well-being and distress with a secondary outcome that rated burnout (physical fatigue, cognitive weariness, tension and listlessness). Participants were randomly allocated to NNBT or CBT and followed up for 12 months. The authors found that participants in both the NNBT and CBT groups reported significantly higher aggregate well-being scores and significantly reduced burnout at the end of treatment and at all subsequent time points. There was no significant difference in outcomes between the two treatments, suggesting they were equally efficacious. In this sense, the trial by Stigsdotter et al is an important contribution to our understanding about the potential role of using nature gardens to treat stress-related illnesses. By using a randomised controlled design the authors addressed the perennial problem of confounding which plagues much research into the impact of the natural environment on health. Additionally, by measuring outcomes over the long term, this study offers insight into the sustainability of health gains achieved by exposure to a nature garden. However, it is too premature to claim off the back of this one study that nature gardens should underpin evidencebased guidelines for managing stress-related illnesses. It remains uncertain what the added value of exposure to nature is over and above the contribution made by CBT or exercises in mindfulness, given that this study was not designed as a superiority trial. Furthermore, the recruited population were predominantly well-educated females and so findings may be prone to forms of performance and selection bias. This could be minimised in future evaluations by adopting a more pragmatic design that includes a broader range of patients who attend primary care with stress-related illnesses, including those with physical comorbidities. As acknowledged by the authors, the nature garden is a complex intervention and, although seemingly efficacious, we are left to puzzle how it might work. The concept of an integrated healing environment, which purposefully brings together elements of the built and natural environment to create a therapeutic landscape, has proved successful among people attending cancer support centres. ${ }^{10}$ Nature gardens similarly draw on theory and practice in landscape architecture and it would be instructive to identify the optimal design characteristics likely to restore attention and restoration and reduce stress. As such, future work on nature gardens should also include process evaluations to understand the relationships between intervention theory and health outcomes and how these relationships are potentially mediated by the content of the intervention and how it is delivered, and also by participant responses to the intervention.

\section{Conclusion}

Nature gardens offer a promising alternative to conventional psychological therapies such as CBT for managing stress-related illnesses. There is scope to extend their reach to populations with other common mental health problems such as depression and anxiety, including those with physical long-term conditions. To fully harness the potential of nature gardens for mental health, we need to establish their effectiveness in large pragmatic trials that use active controls and build in process evaluations to better understand how the intervention works and under what conditions.

\section{Peter A. Coventry; Piran C.L. White}

Correspondence: Peter A. Coventry, Department of Health Sciences, Faculty of Science, ARRC Building, University of York, York Y010 5DD, UK. Email: peter.coventry@ york.ac.uk

First received 2 Feb 2018, accepted 28 Mar 2018

\section{References}

1 Wiegner L, Hange D, Bjorkelund C, Ahlborg Jr G. Prevalence of perceived stress and associations to symptoms of exhaustion, depression and anxiety in a working age population seeking primary care-an observational study. BMC Fam Pract 2015; 16: 38

2 Arends I, Bruinvels DJ, Rebergen DS, Nieuwenhuijsen K, Madan I, NeumeyerGromen A, et al. Interventions to facilitate return to work in adults with adjustment disorders. Cochrane Database Syst Rev 2012; 12: CD006389.

3 Naylor C, Das P, Ross S, Honeyman M, Thompson J, Gilburt H. Bringing Together Physical and Mental Health. A New Frontier for Integrated Care. King's Fund, 2016.

4 Mitchell R, Popham F. Effect of exposure to natural environment on health inequalities: an observational population study. Lancet 2008; 372: 1655-60.

5 Defra. A Green Future: Our 25-year Plan to Improve the Environment. HM Government, 2018

6 Bowler DE, Buyung-Ali LM, Knight TM, Pullin AS. A systematic review of evidence for the added benefits to health of exposure to natural environments. BMC Public Health 2010; 10: 456.

7 Gidlow CJ, Jones MV, Hurst G, Masterson D, Clark-Carter D, Tarvainen MP, et al. Where to put your best foot forward: psycho-physiological responses to walking in natural and urban environments. J Environ Psychol 2016; 45: 22-9.

8 Annerstedt M, Wahrborg P. Nature-assisted therapy: systematic review of controlled and observational studies. Scand Journal Public Health 2011; 39: 371-88.

9 White PC, Wyatt J, Chalfont G, Bland JM, Neale C, Trepel D, et al. Exposure to nature gardens has time-dependent associations with mood improvements for people with mid- and late-stage dementia: innovative practice. Dementia (London) 2017: 1471301217723772

10 Butterfield A, Martin D. Affective sanctuaries: understanding Maggie's as therapeutic landscapes. Landscape Res 2016; 41: 695-706. 